\title{
Monitoring of Bridges by MT-InSAR and Unsupervised Machine Learning Clustering Techniques
}

\author{
Valerio Gagliardi*a, Fabio Tosti ${ }^{\mathrm{b}}$, Luca Bianchini Ciampoli ${ }^{\mathrm{a}}$, Fabrizio D’Amico ${ }^{\mathrm{a}}$, Amir M. Alani ${ }^{\mathrm{b}}$, \\ Maria Libera Battaglierec ${ }^{c}$, Andrea Benedetto ${ }^{a}$ \\ ${ }^{a}$ Department of Engineering, Roma Tre University, Via Vito Volterra 62, 00146, Rome, Italy \\ ${ }^{\mathrm{b}}$ School of Computing and Engineering, University of West London (UWL), St Mary's Road, \\ Ealing, London W5 5RF, UK \\ ${ }^{c}$ Italian Space Agency (ASI), Via del Politecnico, 00133, Rome, Italy
}

\begin{abstract}
Continuous monitoring of critical infrastructures is crucial to prevent catastrophic events such as collapse of viaducts and prioritising maintenance interventions. However, developing effective monitoring approaches must rely on the collection of numerous information, such as the time series of structural deformations. In this context, various groundbased non-destructive testing (NDT) methods have been used in monitoring the structural integrity of transport infrastructures. These require routine and systematic application at the network level over long periods of time to build up a solid database of information, involving many efforts from stakeholders and asset owners in the sector. To this effect, satellite-based remote sensing techniques, such as the Multi-temporal Interferometric Synthetic Aperture Radar (MT-InSAR), have gained momentum due to the provision of accurate cumulative structural displacements in bridges. Although the application of the InSAR monitoring technique is established, this is limited by the high amount of time required for the interpretation of data with high spatial and temporal density. This research aims to demonstrate the viability of MT-InSAR techniques for the structural assessment of bridges and the monitoring of damage by structural subsidence, using high-resolution SAR datasets, integrated with complementary Ground-Based (GB) information. To this purpose, high-resolution SAR dataset of the COSMO-SkyMed (CSK) mission provided by the Italian Space Agency (ASI), were acquired and processed in the framework of the ASI-Open Call approved Project "MoTiB" (ID 742). To elaborate, a Persistent Scatterer Interferometry (PSI) analysis is applied to identify and monitor the structural displacements at the Rochester Bridge, in Rochester, Kent, UK. To explore the viability of Machine Learning algorithms in detecting critical scenarios in the monitoring phases, an Unsupervised ML Clustering approach, which generates homogeneous and well-separated clusters, is implemented. Each PS data-point is allocated to specific cluster groups, based on individual deformation trend features and the values of displacements from the historical time-series. This research paves the way for the development of a novel interpretation approach relying on the integration between remote-sensing technologies and on-site surveys to improve upon current maintenance strategies for bridges and transport assets.
\end{abstract}

Keywords: Remote Sensing, COSMO-SkyMed, Persistent Scatterers Interferometry, Non-Destructive Assessment, Transport Infrastructure Monitoring, InSAR, X-Band, Satellite Remote Sensing.

\section{INTRODUCTION}

Monitoring the conditions of transport infrastructures, such as railways, roads and bridges, is a priority for asset owners and administrators to ensure structural stability, operational safety and to prevent damage and deterioration - leading to expensive rehabilitation - or even failures or collapses [1].

*valerio.gagliardi@uniroma3.it; phone +39 06 57333617; 
Currently, several on-site non-destructive testing (NDT) technologies and sensors are available for subsidence monitoring and displacement mapping. Amongst others, accelerometers [2], strain gauges [3], Global Position System (GPS), levelling [4-5], Ground Penetrating Radar (GPR), Infrared Thermography (IRT) [6-10] and terrestrial SAR Interferometry [11], are recognised as viable technologies for infrastructure monitoring.

However, on-site surveys are costly and are difficult to implement at the network level due to economic and administrative budget constraints. To overcome this limitation, several innovative satellite-based remote sensing techniques, i.e., the Persistent Scatterers Interferometry (PSI) among which the PS-InSAR [12-13] and the Small BAseline Subset (SBAS) [14], have gained momentum in the last few years for the monitoring of transport assets and the investigation of nearby areas.

a)

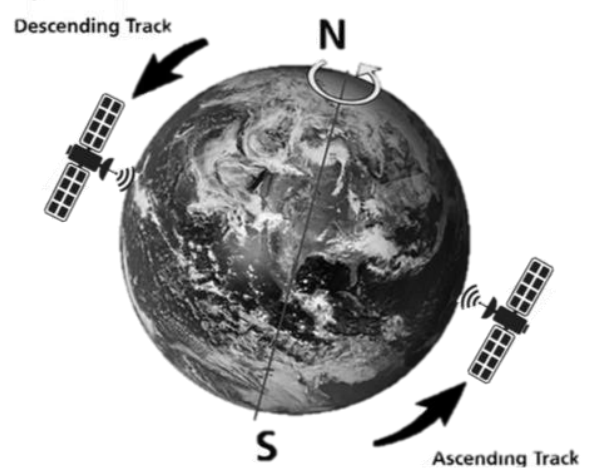

b)

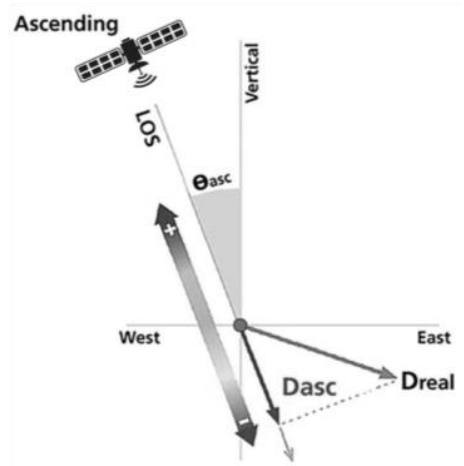

Figure 1: a) SAR data acquisition along an Ascending and a Descending orbit; b) the displacement $D_{\text {asc }}$ measured by the SAR is a component of the real displacement $D_{\text {real }}$

\section{AIMS AND OBJECTIVES}

This research aims at investigating the viability of using Machine Learning (ML) Clustering Techniques, to automatically elaborate large number of information from historical PS time-series, with the purpose to gain useful information for the results interpretation stage. Within this context, this study reports a ML approach, based on the use of the high-resolution COSMO-SkyMed (X-Band) SAR imagery to facilitate a more effective management of linear transport infrastructures.

\section{METHODOLOGY}

\subsection{Multi-Temporal InSAR for Transport Infrastructure Monitoring}

The working framework of the multi-temporal Interferometric Synthetic Aperture Radar (SAR) technique is based on a statistical analysis of the signals emitted by the on-satellite sensor and back-scattered by a network of coherent targets on the ground, i.e., the Persistent Scatterers (PSs). This approach allows to estimate the displacements occurred between different acquisitions by a separation between the phase shift related to the ground motions and the phase component due to the atmosphere, the topography and the signal noise contributions [13,14]. An advantage of these techniques is the relatively lighter data-processing required for the assessment of displacements and the detection of critical areas, as opposed to the higher computational load needed in other approaches. Therefore, the MT-InSAR approach has proven ideal in monitoring transport infrastructures, as the high density of radar stable targets allows for more accurate measurements. To this effect, several scientific contributions related to this application of the PSI techniques can be found in the literature, as reported in Tab. 1. 
Tab. 1 -PSI applications for transport infrastructure monitoring

\begin{tabular}{cc}
\hline Infrastructure type & References \\
\hline Railways & {$[15-21]$} \\
Highways and tunnels & {$[22-24]$} \\
Bridges & {$[25-29]$} \\
Airport runways & {$[30-32]$} \\
\hline
\end{tabular}

This evidence confirms that the use of the above satellite-based remote sensing techniques in these areas of endeavour is established, and they are becoming a popular asset management tool. Furthermore, several studies confirm the potential of applying Machine Learning (ML) algorithms and PSI techniques to assess resilience of infrastructures. [33-35].

\section{EXPERIMENTAL FRAMEWORK}

\subsection{The Rochester Bridge Case Study}

The Rochester Bridge is located in Rochester, Medway (UK) and was for centuries the lowest fixed crossing of the River Medway in the South-East of England. The bridge allows to link the towns of Strood and Rochester in Medway. There have been several generations of bridges at this spot, and the current "bridge" is composed by four separate bridges: the "Old" bridge and the "New" bridge carrying the A2 road, the "Service" bridge carrying service pipes and cables and the "Railway" bridge carrying the railway. Currently, all the bridges are owned and maintained by the Rochester Bridge Trust, except for the railway bridge, which is owned by Network Rail. This nineteenth-century bridge was reconstructed between 1910 and 1914, and arches were installed at their present position above the roadway, to provide a larger clearance for ships movement under the bridge. Between 1965 and 1970, the Rochester Bridge Trust built a second roadway bridge on the piers of the disused railway bridge, immediately downstream from the roadway bridge. The reconstructed Victorian bridge is nowadays known as the "Old Bridge", whereas the second roadway bridge is known as the "New Bridge" [36].

\subsection{The SAR Datasets}

A multi-frequency SAR dataset of X-Band data was collected and processed by the PSI technique to quantify potential displacements on the inspected bridge. For this purpose, the assessment of the area is performed through the processing of higher resolution X-Band SAR images collected by the COSMO-SkyMed satellite mission and operating at a frequency of $9.6 \mathrm{GHz}$ (i.e., corresponding to a wavelength of $3.1 \mathrm{~cm}$ ). This allows to collect data with ground-resolution cells of $3 \times 3 \mathrm{~m}$ and to detect displacements with a millimetre accuracy, under ideal conditions. These products were provided by the Italian Space Agency (ASI) in the framework of the Project MoTIB, ID 742, (COSMO -SkyMed@Open Call for Science), and distributed by the e-Geos group. Table 2 reports the SAR dataset characteristics.

Tab. 2 - SAR dataset characteristics

\begin{tabular}{cc}
\hline Satellite missions & COSMO-SkyMed (ASI) \\
\hline Time Period & $03 / 2017-12 / 2019$ \\
Operating Frequency & X-Band $(9.6 \mathrm{GHz})$ \\
Wavelength & $\lambda=3.1 \mathrm{~cm}$ \\
Range / Azimuth Resolution & $3 \mathrm{~m} / 3 \mathrm{~m}$ \\
Acquisition mode & Stripmap \\
Processing Level & HIMAGE \\
\hline
\end{tabular}




\subsection{The PSI Processing}

The SAR datasets were processed according to the PSI method [12] by means of the Interferometric Stacking Module of the Software SARscape [37] integrated in the Software ENVI, licensed within the framework of the European Space Agency (ESA) approved project "STRAIN2: Sensing TRAnsport INfrastructures 2" (EOhops ID 53071). More specifically, the PSI technique operates by the application of consecutive stages [12-13]. As a result, stable reflectors, i.e., the PSs, can be identified over the inspected area. However, SAR satellites can only detect displacements in the Line-of-Sight $(\mathrm{LoS})$, with reference to a specific orbit-related incident angle.

Therefore, the displacement detected is a component of the real displacement occurring on the ground. Different approaches were proposed to evaluate the real displacements-velocity-vector, from datasets acquired with different acquisition geometries (i.e., Ascending and Descending) [38-39]. For the investigated area of interest, only SAR images acquired in descending geometries of the COSMO-SkyMed mission were available. To this effect, in this work we will refer only to the displacements detected in the LoS-direction of the SAR sensors.

\subsection{The k-means Algorithm}

The k-means algorithm is an unsupervised clustering technique that allows to divide a set of objects into k groups based on their attributes; it yields to satisfying results and it is still widely used as a partition group analysis algorithm [40-41]. A given data set $x$ is grouped into $\mathrm{k}$ a-priori fixed clusters by minimising the total within the cluster variance (i.e. the sum of the squared errors) [40-41].

The clustering process is also composed of competition and adjustment and it has been implemented according to the following steps: i) The centroids $\mu$ of the $\mathrm{K}$ desired clusters $\mathrm{C}=\left(c_{k}, \mathrm{k}=1, \ldots, \mathrm{K}\right)$ were randomly initialised; ii) each data sample $x_{i}$ was assigned to the closest cluster centroid $\mu_{k}$, i.e., with the smallest Euclidean distance. Accordingly, the sum of the squared error (i.e., within the cluster variance) over all clusters is minimised. The function $J(t)$ is the sum of the squared error and $\mu_{k}$ is the cluster centroid of cluster $c_{k}$. [40-42]:

$$
J(C)=\min \left\{\sum_{k=1}^{K} \sum_{x_{i} \varepsilon c_{k}}\left\|x_{i}-\mu_{k}\right\|^{2}\right\}
$$

iii) The cluster centroids were adjusted by a replacement with the mean values of all the associated data samples according to Eq. 2:

$$
\mu_{k}(t+1)=\frac{1}{N_{k}} \sum_{x_{i} \varepsilon c_{k}} x_{i}
$$

where $\mu_{n}(t+1)$ is the cluster centroid of cluster $j$ at time $t+1$ and $N_{k}$ is the number of the data samples in cluster $c_{k}$.

The processing steps ii) and iii) were reiterated until a stabilised condition is reached (i.e., the cluster membership does not change anymore). To avoid convergence to a local minimum, the algorithm was replicated 1000 times and the most frequent result was used. However, without any a-priori information about the expected number of the forest structure types and the class definitions, the a-priori determination of $k$ was not feasible. In order to solve this problem, the optimal quantity of clusters can be determined by means of the within-cluster variance (i.e., within-cluster sum of distances from the data points to the cluster centroids). For several values of $k$ (i.e., different number of clusters) the within-cluster sum of distances was calculated in the replicative clustering process and plotted against $k$. The withincluster sum of distances decreases with increasing values of $k$, as the size of clusters become smaller and thus the variance around the cluster centroids decreases. The point of the curve where the successive decrease in the withincluster sum of distances become significantly smaller identifies the optimal $k$ to describe the data structure.

\section{RESULTS}

The analysis of high-resolution STRIPMAP X-Band COSMO-SkyMed products allowed to obtain several PSs over the entire bridge structure. The outputs were converted in a (.kml) file format, imported into the Google Earth Pro platform 
and displayed as a function of the average velocity vector. The green points are referred to stable scatterers with a displacement velocity ranging from $-4 \mathrm{~mm} / \mathrm{yr}$ and $+4 \mathrm{~mm} / \mathrm{yr}$. Several PSs show a comparable deformation trend and appear to be affected by cyclic down-lifting and up-lifting trends, most likely related to seasonal effects. The set of PSs resulting from the processing of COSMO-SkyMed data in the descending geometry is shown in Fig.3.

a)

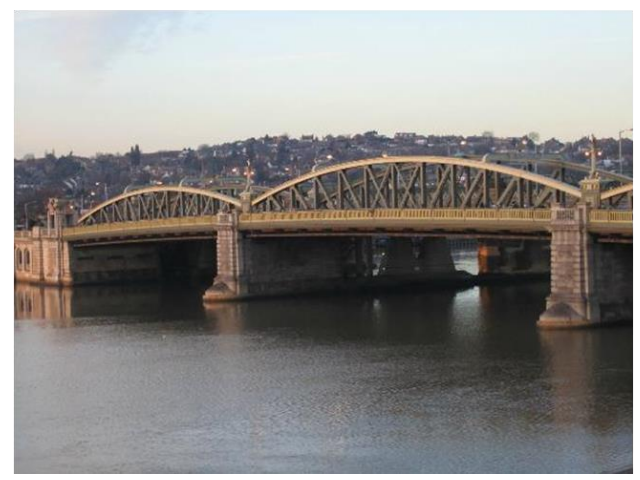

b)

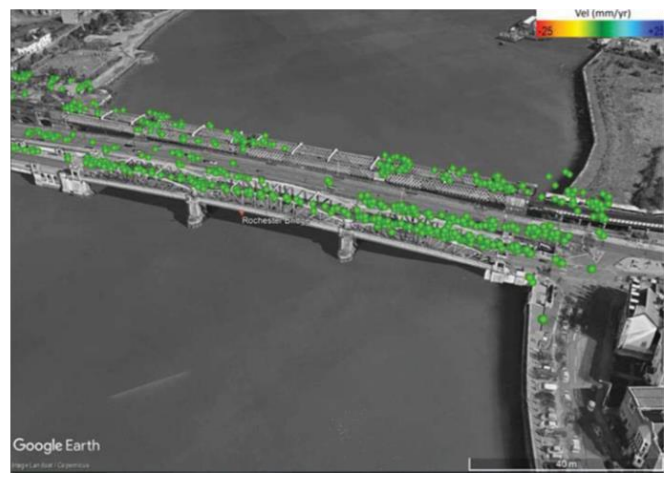

Figure 2: a) Rochster Bridge b) PSI results on the Rochester Bridge. Data display is performed in relation to the average trend of velocity of deformation $(\mathrm{mm} / \mathrm{yr})$

\subsection{Time-series and Clustering Interpretation Operations}

To associate the identified PSs to individual structural elements of the bridge, a clustering operation was performed. To this effect, an Unsupervised ML Clustering approach was adopted to explore the viability of Machine Learning algorithms in detecting critical situations in the monitoring phases. Amongst the others, the k-means ML clustering algorithm was found to be effective in generating homogeneous and distinguished clusters. Each PS data-point is located to specific cluster groups, based on deformation-trend features and the values of displacements from the historical timeseries.

Clustering was perfomed through a built-in Matlab code, determining the $k$ number of clusters. Defining many clusters can erroneously allocate PSs from different structural elements to a same element, whereas using a small number of clusters (i.e., 1 or 2) can gather all PSs as part of a single groups, leading to low statistical power. Before carrying out the procedure with k-means, outliers must be therefore identified and eliminated from the dataset. At this stage of the presented research, the feasibility of the proposed approach was tested by analysing time-series covering three years of observation (2017-2019). Similarly, the displacement trend of the identified PS cluster was investigated over the same time-period.

The dataset is composed by a total of 196 PSs. For each acquisition, the relative position of the PS referred to a stable Ground Control Point (GCP) is known. The GCP is statistically detected and it is located externally from the bridge. This allows to detect millimetre displacements over the investigated time. In Figure 3, the mean values of the millimetre deformations of the PS-clusters are reported over the investigation period (from January 2017 to November 2019).

This allows the identification of comparable sub-clusters of PSs with a similar deformation trend within the investigated time-period. Following this approach, four main sub-cluster patterns are identified by an analysis of the historical deformation time-series. Therefore, the geo-location of sub-cluster patterns over the bridge pier, confirming the presence of different groups of nearby PSs, could serve as a useful tool to indicated different sub-areas of deformation. The identified sub-cluster patterns have shown distinctive deformation movements between October 2018 and May 2018. The outcomes of this operation are reported in Fig. 6, where the location of each sub-cluster pattern at Pier n.2 of the "Road-Bridge" and the corresponding time-series of the deformations are reported. 
a)

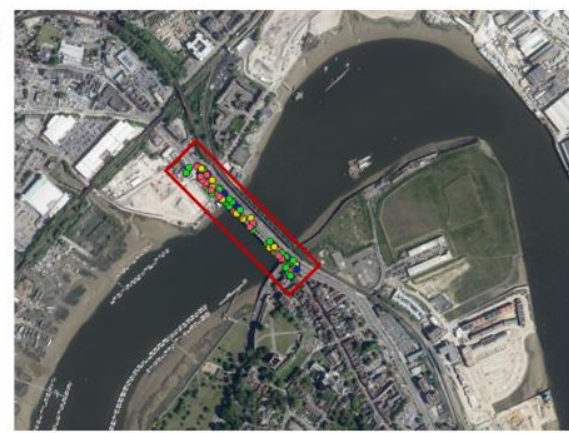

c)

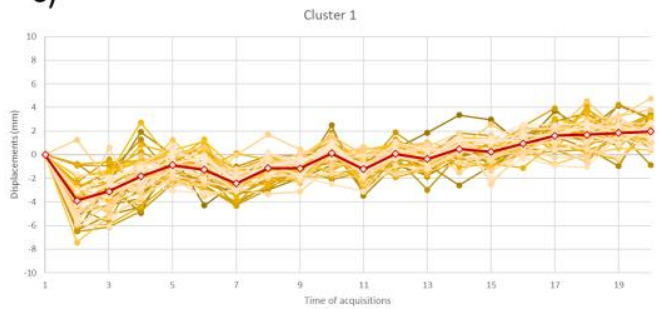

e)

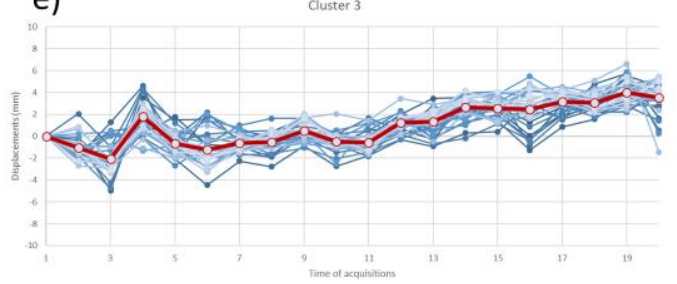

b)

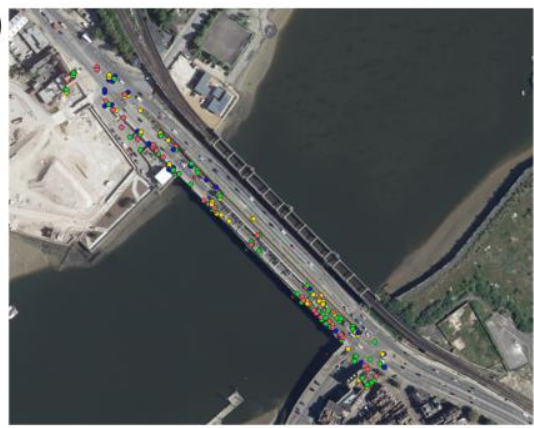

d)

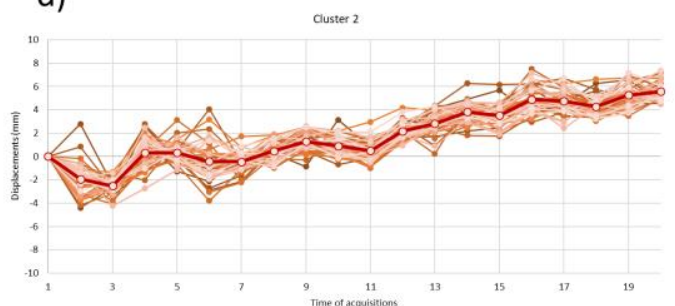

f)

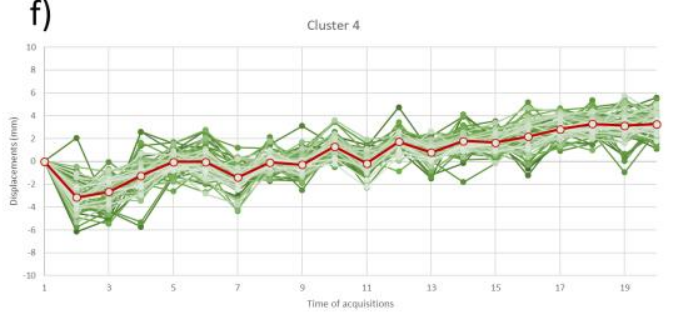

Figure 3: a, b) PS cluster located at the "Road bridge" sorted in sub-cluster points; PS displacements time-series and deformation velocity outputs reconstruction of the c) cluster 1, d) cluster 2, e) cluster 3 and f) cluster 4

Figure 3 (c-f) shows the displacements time-series and the average values of displacements for the identified cluster groups, displayed as a red line. It is observed that all the identified clusters were characterised by an up-lifting trend. The average velocity value for this occurrence is relavely low (i.e., $+0.5 \mathrm{~mm} / \mathrm{yr}$ ). A seasonal deformation trend is also observed in all groups, and it is more remarkable in cluster 1 group.

\section{CONCLUSIONS}

This research aims to demonstrate the viability of the MT-InSAR techniques for the structural assessment of bridges and the monitoring of damage by structural subsidence, using high-resolution SAR datasets. To this purpose, high-resolution SAR dataset of the COSMO-SkyMed (CSK) mission provided by the Italian Space Agency (ASI), were acquired and processed in the framework of the ASI-Open Call approved Project "MoTiB" (ID 742). A Persistent Scatterer Interferometry (PSI) analysis is applied to identify and monitoring the structural displacements at the Rochester Bridge, in Rochester, Kent, UK. To explore the viability of Machine Learning clustering algorithms in detecting critical situations in the monitoring phases, an Unsupervised ML Clustering approach, which generates homogeneous and wellseparated clusters, is implemented. Each PS data-point is located to specific cluster groups, based on the deformationtrend and the values of displacements of the historical time-series. A comparison between the results of data processed establishes the viability of using ML algorithms to process several amounts of PSs which are typically related to highresolution X-band SAR data, and sets novel perspectives in the monitoring of transport infrastructures. This information is fundamental to improve upon the current capacity of pavement management systems (PMSs) to predict critical displacements and optimise maintenance interventions prior to any structural failure. Sorting the PSs through a 
clustering operation was found effective in terms of the time required for the interpretation of the results, paving the way for a future implementation of this approach.

This information is of interest for a potential integration of these outcomes with complementary on-site investigations (e.g., levelling or GPR) on critical areas identified preliminarily by the PSI technique. Furthermore, this approach could allow for a more effective planning of preventive maintenance on the bridge, to limit full rehabilitation activities.

This research paves the way for the development of a novel interpretation approach relying on the integration between remote-sensing technologies and on-site surveys to improve upon current maintenance strategies for bridges and transport assets.

\section{ACKNOWLEDGMENTS}

This research is carried out using COSMO-SkyMed Products- CSK @ C ASI (Italian Space Agency), delivered under an ASI licence to use in the framework of COSMO-SkyMed Open Call for Science. In addition, the Authors would like to acknowledge and thank the Rochester Bridge Trust for facilitating and supporting this research. This research is supported by the Italian Ministry of Education, University and Research under the National Project "EXTRA TN", PRIN 2017, Prot. 20179BP4SM. In addition, the authors acknowledge funding from the MIUR, in the frame of the "Departments of Excellence Initiative 2018-2022", attributed to the Department of Engineering of Roma Tre University.

\section{REFERENCES}

[1] Chang P.C., Flatau A. \& Liu S. C. "Review Paper: Health Monitoring of Civil Infrastructure". Structural Health Monitoring, 2(3), 257-267. 2003.

[2] Kongyang C., Mingming L., Xiaopeng F., Mingming W. and Wu J., "Road condition monitoring using onboard Three-axis Accelerometer and GPS Sensor," 6th International ICST Conference on Communications and Networking in China, Harbin, 2011

[3] Olund J., \& DeWolf J., "Passive Structural Health Monitoring of Connecticut's Bridge Infrastructure". Journal of Infrastructure Systems, 13(4), 330-339. 2007.

[4] Mossop A., Segall P., "Subsidence at The Geysers Geothermal Field, N. California from a comparison of GPS and leveling surveys". Geophys. Res. Lett., 24, 1839-1842, 1997

[5] Sato H.P., Abe K., Ootaki O., "GPS-measured land subsidence in Ojiya City, Niigata Prefecture, Japan”. Eng. Geol., 67, 379-390, 2003.

[6] Saarenketo T. \& Scullion T., (2000). "Road evaluation with ground penetrating radar". Journal of Applied Geophysics, 43(2-4), 119-138.

[7] Lagüela S., Solla M., Puente I., \& Prego F. J., (2018). "Joint use of GPR, IRT and TLS techniques for the integral damage detection in paving". Construction and Building Materials, 174, 749-760.

[8] Bianchini Ciampoli L., Artagan S. S., Tosti F., Gagliardi V., Alani A. M. and Benedetto A., "A comparative investigation of the effects of concrete sleepers on the GPR signal for the assessment of railway ballast", $17^{\text {th }}$ International Conference on Ground Penetrating Radar (GPR), Rapperswil, pp. 1-4, doi: 10.1109/ICGPR.2018.8441588, 2018.

[9] Benedetto, F. Tosti, L. B. Ciampoli, and F. D'Amico, "GPR applications across engineering and geosciences disciplines in Italy: A review,” IEEE J. Sel. Topics Appl. Earth Obs. Remote Sens., vol. 9, no. 7, pp. $2952-$ 2965, Jul. 2016.

[10] Tosti, F. and Benedetto, A. (2012) "Pavement Pumping Prediction Using Ground Penetrating Radar. Procedia Social and Behavioral Sciences", 53, 1045-1054.https://doi.org/10.1016/j.sbspro.2012.09.954

[11] Mazzanti P. et al. (2015). "Terrestrial SAR Interferometry Monitoring of Natural Slopes and Man-Made Structures". Engineering Geology for Society and Territory - Volume 5. Springer, Cham

[12] Ferretti A., Prati C., Rocca F., "Permanent scatters in SAR interferometry". IEEE Trans Geosci Remote Sens 39(1):8-20, 2001. https ://doi.org/10.1109/36.898661

[13] Ferretti A., Prati C., Rocca F. "Nonlinear subsidence rate estimation using permanent scatterers in differential SAR interferometry”. IEEE Trans Geosci Remote Sens 38(5):2202-2212, 2000.

[14] Lanari R., Mora O., Manunta M., Mallorqui J.J., Berardino P., Sansosti E. "A small baseline approach for investigating deformation on full resolution differential SAR interferograms". IEEE Trans. Geosci. Remote Sens. 42, 1377-1386, 2004. 
[15] Bianchini Ciampoli L., Gagliardi V., Clementini C., et al. "Transport Infrastructure Monitoring by InSAR and GPR Data Fusion”. Surv Geophys 41, 371-394. 2020. https://doi.org/10.1007/s10712-019-09563-7

[16] Chang L., Dollevoet R. P., \& Hanssen R. F., "Nationwide Railway Monitoring Using Satellite SAR Interferometry". IEEE Journal of Selected Topics in Applied Earth Observations and Remote Sensing, 10(2),596-604. 2017.

[17] D'Amico F., Gagliardi V., Bianchini Ciampoli L., Tosti F., "Integration of InSAR and GPR Techniques for Monitoring Transition Areas in Railway Bridges". NDT\&E International. 2020.

[18] Qin X., Liao M., Zhang L., \& Yang M.," Structural Health and Stability Assessment of High-Speed Railways via Thermal Dilation Mapping with Time-Series InSAR Analysis". IEEE Journal of Selected Topics in Applied Earth Observations and Remote Sensing, 10(6), 2999-3010. 2017.

[19] Gagliardi V., Bianchini Ciampoli L., D'Amico F., Alani A. M., Tosti F., Battagliere M. L., Benedetto A., "Bridge monitoring and assessment by high-resolution satellite remote sensing technologies", Proc. SPIE 11525, SPIE Future Sensing Technologies. 2020. doi: 10.1117/12.2579700

[20] Yang Z., Schmid F., Roberts C., Assessment of railway performance by monitoring land subsidence. In: 6th IET conference on railway condition monitoring (RCM 2014), pp 1-6. 2014 https ://doi. org/10.1049/cp.2014.1000

[21] Tosti F., Gagliardi V., D'Amico F., Alani A.M., "Transport infrastructure monitoring by data fusion of GPR and SAR imagery information".Transp Res Proc 2020; 45:771-778. 721. https://doi.org/10.1016/j.trpro.2020.02.097

[22] Koudogbo F., Urdiroz A., Robles JG., Chapron G., Lebon G., Fluteaux V., Priol G. "Radar interferometry as an innovative solution for monitoring the construction of the Grand Paris Express metro network-first results". In: World tunnel conference, 2-25 April, Dubai, 2018

[23] Barla G. et al., "InSAR monitoring of tunnel induced ground movements. Geomechanik und Tunnelbau" 9(1):15-22, 2016.

[24] Perissin D., Wang Z., \& Lin H., (2012). "Shanghai subway tunnels and highways monitoring through CosmoSkyMed Persistent Scatterers". ISPRS Journal of Photogrammetry and Remote Sensing, 73, 58-67.

[25] Alani A. M., Tosti F., Bianchini Ciampoli L., Gagliardi V., Benedetto A., (2020). "An integrated investigative approach in health monitoring of masonry arch bridges using GPR and InSAR technologies". NDT \& E International, 102288. https://doi.org/10.1016/j.ndteint.2020.102288

[26] Gagliardi V., Benedetto A., Bianchini Ciampoli L., D'Amico F., Alani A. M., Tosti F. "Health monitoring approach for transport infrastructure and bridges by satellite remote sensing Persistent Scatterers Interferometry (PSI)", Proc. SPIE 11534. 2020. https://doi.org/10.1117/12.2572395

[27] Jung J., Kim D-J, Palanisamy Vadivel SK, Yun S-H. "Long-term deflection monitoring for bridges using X and C-band time-series SAR interferometry". Remote Sens 2019;11(11):1258. 715

[28] Bianchini Ciampoli L., Gagliardi V., Calvi A., D’Amico F., Tosti F. "Automatic network-level bridge monitoring by integration of InSAR and GIS catalogues". Proceedings of SPIE 2019. https://doi.org/10.1117/12.2527299

[29] V. Gagliardi, L. Bianchini Ciampoli, F. D’Amico, A. M. Alani, F. Tosti, A. Benedetto, "Multi-Temporal SAR Interferometry for Structural Assessment of Bridges: The Rochester Bridge Case Study". International Airfield and Highway Pavements Conference 202. https://ascelibrary.org/doi/abs/10.1061/9780784483510.028

[30] Gao M., Gong H., Chen B., Zhou C., Chen W., Liang Y., Shi M., Si Y., (2016). "InSAR time-series investigation of long-term ground displacement at Beijing Capital International Airport, China". Tectonophysics, 691, 271-281.

[31] Bianchini Ciampoli L., Gagliardi V., Ferrante C., Calvi A., D’Amico F., Tosti F., "Displacement Monitoring in Airport Runways by Persistent Scatterers SAR Interferometry". Remote Sens. (2020), 12, 3564. https://doi.org/10.3390/rs12213564

[32] Gagliardi, V.; Bianchini Ciampoli, L.; Trevisani, S.; D’Amico, F.; Alani, A.M.; Benedetto, A.; Tosti, F. "Testing Sentinel-1 SAR Interferometry Data for Airport Runway Monitoring: A Geostatistical Analysis". Sensors 2021, 21, 5769. https://doi.org/10.3390/s21175769

[33] Fiorentini N., Maboudi M., Leandri P., Losa M. "Can Machine Learning and PS-InSAR Reliably Stand in for Road Profilometric Surveys? “. Sensors 2021, 21, 3377. https://doi.org/10.3390/s21103377

[34] Fadhillah M.F., Achmad A.R., Lee C.-W. "Integration of InSAR Time-Series Data and GIS to Assess Land Subsidence along Subway Lines in the Seoul Metropolitan Area, South Korea". Remote Sens. 2020, 12, 3505. https://doi.org/10.3390/rs12213505 
[35] Fiorentini N., Maboudi M., Losa M., and Gerke M. “Assessing Resilience of Infrastructures Towards Exogenous Events by Using Ps-Insar-Based Surface Motion Estimates and Machine Learning Regression Techniques", ISPRS Ann. Photogramm. Remote Sens. Spatial Inf. Sci., V-4-2020, 19-26, https://doi.org/10.5194/isprs-annals-V-4-2020-19-2020, 2020

[36] Rochester Bridge Trust, Internet resource accessed (20 August 2021) at https://rbt.org.uk/bridges

[37] SARscape technical description, 2012. Internet resource accessed 2020-12-28 at http://www.sarmap.ch/pdf/SARscapeTechnical.pdf

[38] Dalla Via G., Crosetto M., and Crippa B. "Resolving vertical and east-west horizontal motion from differential interferometric synthetic aperture radar: The L'Aquila earthquake. Journal of geophysical research: solid earth "117, no. B2.2012.

[39] Gagliardi V., Bianchini Ciampoli L., D'Amico F., Tosti F., Alani A. and Benedetto A. "A Novel GeoStatistical Approach for Transport Infrastructure Network Monitoring by Persistent Scatterer Interferometry (PSI)". In: 2020 IEEE Radar Conference, Florence, Italy, 2020, pp. 1-6, doi: 10.1109/RadarConf2043947.2020.9266336

[40] MacQueen J., 1967. "Some methods for classification and analysis of multivariate observations". Proceedings of the Fifth Berkeley Symposium on Mathematical Statistics and Probability, 1: Statistics, 281-297.

[41] Jain K.A., 2010. "Data clustering: 50 years beyond K-Means”. Pattern Recognit. Lett. 31 (8), 651-666.

[42] Xu R., Wunsch, D., 2005. "Survey of clustering algorithms”. IEEE Trans. Neural Netw.16 (3), 645-678 